\title{
Detection of lymph node micrometastasis in pNO early gastric cancer: Efficacy of duplex RT-PCR with MUC2 and TFF1 in mucosal cancer
}

\author{
HIROMICHI SONODA ${ }^{1}$, KAZUO YAMAMOTO ${ }^{2}$, RYOJI KUSHIMA ${ }^{3}$, HIROSHI YAMAMOTO ${ }^{1}$, \\ HIROYUKI NAITOH $^{1}$, HIDETOSHI OKABE ${ }^{2}$ and TOHRU TANI ${ }^{1}$ \\ Departments of ${ }^{1}$ Surgery, ${ }^{2}$ Clinical Laboratory Medicine, and ${ }^{3}$ Clinical Pathology, \\ Shiga University of Medical Science, Shiga, Japan
}

Received January 30, 2006; Accepted March 31, 2006

\begin{abstract}
We previously reported that MUC2 is a useful marker in the detection of lymph node micrometastasis (LMM) in gastric cancer. To improve the detection rate, we focused on a TFF1 gene. We used the duplex reverse transcriptasepolymerase chain reaction (RT-PCR) method with MUC2 and TFF1 genes to detect LMM in histologically node negative (pNO) early gastric cancer (EGC) and evaluated their effectiveness. A total of 310 lymph nodes from 33 patients with pNO EGC were analyzed. All carcinoma specimens were positive for MUC2 and/or TFF1. The positive rate of TFF1 was significantly higher than MUC2 in the undifferentiated carcinoma specimens $(\mathrm{p}=0.002)$. The detection rate of duplex RT-PCR with MUC2 and TFF1 was higher than that of MUC2 or TFF1 alone. In mucosal cancer, 7 cases were positive for duplex RT-PCR. Of these 7 cases, 3 were MUC2positive/TFF1-negative while 4 were MUC2-negative/TFF1positive. LMMs were not detected in elevated-type primary mucosal cancers with a 20-30-mm diameter. The duplex RTPCR assay with both MUC2 and TFF1 provides a higher LMM detection rate than either MUC2 or TFF1 alone, especially in mucosal gastric cancer. LMM detected in this manner may prove useful in broadening the indications for endoscopic mucosal resection in elevated-type cancer.
\end{abstract}

\section{Introduction}

Regional lymph node metastasis is the most important prognostic factor for patients with gastric cancer. Therefore, radical gastrectomy with D2 lymph node dissection is recognized as

Correspondence to: Dr Hiromichi Sonoda, Department of Surgery, Shiga University of Medical Science, Seta Tsukinowa-cho, Ohtsu, Shiga 520-2192, Japan

E-mail: hirosono@belle.shiga-med.ac.jp

Key words: duplex RT-PCR, MUC2, TFF1, micrometastasis the standard surgical procedure for treating early gastric cancer (EGC). Lymph node metastasis in EGC comprises up to $4 \%$ of the mucosal type and approximately $23 \%$ of the submucosal type (1). To increase the quality of life of patients with EGC, minimally invasive procedures, such as endoscopic mucosal resection (EMR), laparoscopic surgery and a reduced form of a lymphadenectomy are preferred treatment options. The recently developed EMR procedure, endoscopic submucosal dissection (ESD), makes en bloc resection of large lesions possible (2-6). Thus, it is more important to select those patients who are void of lymph node metastatic disease.

Lymph node micrometastasis (LMM) is defined as micrometastatic foci that cannot be detected by H\&E staining (7). Micrometastases that exist at the time of the primary tumor operation are thought to be involved in tumor recurrence $(8,9)$. Carcinoembryonic antigen (CEA) and cytokeratins (CK) have been widely used to detect LMM in gastric and colorectal cancer (7,10-21). These CEA and CK assays, however, have high false positive rates (22-26). Therefore, we hope to identify another target molecule that effectively detects LMM in gastric cancer. We previously reported that MUC2 is a useful marker for detecting LMM in gastric cancer (27), but it is rarely expressed in early, poorly differentiated adenocarcinomas. To improve the detection rate of LMM in gastric cancer, we focused on a TFF1 gene which is preferentially expressed in diffuse-type gastric cancer cells (28). We developed simultaneous detection methods of MUC2 and TFF1 genes by using duplex RT-PCR, and achieved high gastric cancer LMM detection rates.

\section{Materials and methods}

Patients. We selected 33 histologically node negative (pN0) EGC patients who underwent curative surgery in our surgery department between July 2002 and June 2004. This study followed the ethical standards of the Helsinki Declaration and the patients gave written informed consent according to our hospital's criteria. The study group consisted of 19 men (57\%) and 14 women (43\%), where the mean age was 62 years (range, 33-81 years). Pathological diagnosis and classification were based on guidelines from the Japanese Gastric Cancer Association (29). 
Table I. MUC2 and TFF1 gene expression in primary gastric cancer lesions.

\begin{tabular}{ccc}
\multicolumn{2}{c}{ Positive rate } \\
\hline MUC2 & TFF1 & Duplex marker \\
& & (MUC2 + TFF1)
\end{tabular}

Differentiated carcinoma

$85 \%$

(17 cases/20 cases)

Undifferentiated carcinoma

$38.5 \%$

( 5 cases $/ 13$ cases $)$

$\underset{\mathrm{p}=0.002}{(13 \text { cases } / 13 \text { cases })}$

(17 cases/20 cases)

$100 \%$

$90.9 \%$

$100 \%$

Total

$66.7 \%$

(30 cases/33 cases)

(33 cases/33 cases)

Differentiated carcinoma, well to moderately differentiated adenocarcinoma; undifferentiated carcinoma, poorly differentiated adenocarcinoma and signet ring cell carcinoma.

Carcinomas and lymph node samples. A total of 33 primary gastric tissues and 310 lymph nodes were obtained from 33 patients. Fourteen lymph nodes from patients with nonneoplastic diseases, such as peptic ulcers, ulcerative colitis, and cholecystolithiasis, were used as negative controls. Each lymph node was cut into two pieces. One piece was formalinfixed and paraffin-embedded for histological examination, while the other was kept at $-80^{\circ} \mathrm{C}$ until RNA extraction. Sterile instruments and gloves were used to dissect the primary tumors and lymph nodes from the omentum to avoid contamination.

Histological examination. The paraffin-embedded sections were stained with H\&E. These sections were then examined independently by two experienced pathologists (R.K. and H.O.), blind to the molecular data.

RNA extraction. Carcinoma tissues and lymph node samples were homogenized with an RNase-free disposable homogenizer (Scientific Specialties, CA, USA). Total cellular RNA was extracted using TRI Reagent (Molecular Research Center, Ohio, USA), according to the manufacturer's instructions. To ensure the elimination of genomic DNA, each microgram of total RNA was incubated with 1 unit of DNase I (Ambion, Cambridgeshire, UK) at $37^{\circ} \mathrm{C}$ for $30 \mathrm{~min}$. Purified total cellular RNA was quantified and assessed for purity by UV spectrophotometry.

Duplex RT-PCR with MUC2 and TFF1. Prior to PCR, $1.0 \mu \mathrm{g}$ of total RNA was reverse transcripted using random hexamers and Moloney murine leukemia virus reverse transcriptase (First-Strand cDNA synthesis kit, Amersham Pharmacia Biotech, Uppsala, Sweden), as previously described (27). PCR was performed with $0.5 \mu 1$ of reverse transcripted RNA mixture in a final volume of $30 \mu 1$. The amplification reaction mixture contained 3.0 U of Taq polymerase hot start version (Takara, Shiga, Japan), 1.5 U 10X Ex Taq buffer, and $2.5 \mathrm{mM}$ dNTP mixture. The PCR cDNA products of MUC2, TFF1, and $B$-actin were 189,438 , and 838 bp, respectively. The annealing temperature and cycles for duplex PCR were: one cycle of denaturing at $94^{\circ} \mathrm{C}$ for $2 \mathrm{~min}$, followed by 35 cycles $\left(94^{\circ} \mathrm{C}\right.$ for $15 \mathrm{sec}, 60^{\circ} \mathrm{C}$ for $45 \mathrm{sec}$, and $72^{\circ} \mathrm{C}$ for $\left.3 \mathrm{~min}\right)$. The primer sequences were 5'-CCATTCTCAACGACAACCCC TACTACCCC-3' and 5'-TCCAATGGGAACATCAGG ATACATGGTGGC-3' for MUC2, and 5'-TTTGGAGCAGA GAGGAGG-3' and 5'-TTGAGTAGTCAAAGTCAGAGC AG-3' for TFF1. Aliquots $(7 \mu \mathrm{l})$ from each reaction mixture were size fractionated on $2 \%$ agarose gel and visualized with ethidium bromide staining. To ensure that the RNA was not degraded, a PCR assay with primers specific for $B$-actin cDNA was performed. The PCR conditions were: one cycle of denaturing at $94^{\circ} \mathrm{C}$ for $5 \mathrm{~min}$, followed by 25 cycles $\left(94^{\circ} \mathrm{C}\right.$ for $1 \mathrm{~min}, 72^{\circ} \mathrm{C}$ for $1 \mathrm{~min}$ ) before final extension at $72^{\circ} \mathrm{C}$ for $7 \mathrm{~min}$. The primer sequences were: $5^{\prime}$-ATCTGGCACCACA CTTGTACAATGAGCTG-3' and 5'-CGTCATACTCCTGC TTGCTGATCCACATCT-3'.

Statistical analysis. Statistical comparisons for significance between different groups were evaluated by the Chi-square test. P-values $<0.05$ were considered statistically significant.

\section{Results}

Expression of MUC2 and TFF1 mRNA in primary gastric carcinoma lesions and control lymph nodes. MUC2 and TFF1 transcripts were expressed in 22 of $33(66.7 \%)$ and 30 of $33(90.9 \%)$ gastric carcinoma specimens, respectively. All gastric carcinoma specimens were positive using the duplex RT-PCR assay (Table I). Alternatively, all of the control lymph nodes were negative for both MUC2 mRNA and TFF1 mRNA.

Correlation between duplex marker (MUC2 and TFF1) expression and tumor histology. MUC2 was expressed in 17 of 20 differentiated carcinomas (well to moderately differentiated adenocarcinoma), 4 of 7 signet ring cell carcinomas, and 1 of 6 
Table II. Clinical features of mucosal gastric cancer patients.

\begin{tabular}{|c|c|c|c|c|c|c|c|c|}
\hline \multirow[t]{2}{*}{ Patient } & \multirow[t]{2}{*}{$\begin{array}{l}\text { Age and } \\
\quad \text { sex }\end{array}$} & \multirow[t]{2}{*}{$\begin{array}{l}\text { Tumor } \\
\text { location }\end{array}$} & \multirow[t]{2}{*}{$\begin{array}{l}\text { Macroscopic } \\
\text { type }\end{array}$} & \multirow[t]{2}{*}{$\begin{array}{l}\text { Tumor } \\
\text { size }(\mathrm{mm})\end{array}$} & \multirow[t]{2}{*}{$\begin{array}{l}\text { Histological- } \\
\text { type }\end{array}$} & \multicolumn{3}{|c|}{$\begin{array}{l}\text { Gene expression of mRNA } \\
\text { in the resected lymph nodes }\end{array}$} \\
\hline & & & & & & MUC2 & TFF1 & $\begin{array}{r}\text { Duplex marker } \\
(\mathrm{MUC} 2+\mathrm{TFF} 1)\end{array}$ \\
\hline 1 & $79 \mathrm{f}$ & $\mathrm{L}$ & depressed & 30 & well & + & - & + \\
\hline 2 & $43 \mathrm{f}$ & M & depressed & 25 & poor & - & + & + \\
\hline 3 & $71 \mathrm{~m}$ & $\mathrm{M}$ & depressed & 30 & poor & + & - & + \\
\hline 4 & $52 \mathrm{~m}$ & M & depressed & 90 & poor & - & + & + \\
\hline 5 & $45 \mathrm{~m}$ & M & depressed & 45 & sig & - & + & + \\
\hline 6 & $69 \mathrm{~m}$ & $\mathrm{~L}$ & depressed & 27 & $\bmod$ & + & - & + \\
\hline 7 & $81 \mathrm{~m}$ & $\mathrm{~L}$ & elevated & 15 & well & - & + & + \\
\hline 8 & $72 \mathrm{~m}$ & $\mathrm{~L}$ & elevated & 20 & well & - & - & - \\
\hline 9 & $47 \mathrm{f}$ & M & elevated & 25 & $\bmod$ & - & - & - \\
\hline 10 & $76 \mathrm{f}$ & M & elevated & 24 & poor & - & - & - \\
\hline 11 & $33 \mathrm{~m}$ & M & depressed & 27 & sig & - & - & - \\
\hline 12 & $76 \mathrm{~m}$ & $\mathrm{U}$ & elevated & 25 & well & - & - & - \\
\hline 13 & $75 \mathrm{f}$ & M & elevated & 22 & well & - & - & - \\
\hline 14 & $55 \mathrm{~m}$ & $\mathrm{M}$ & depressed & 15 & well & - & - & - \\
\hline 15 & $62 \mathrm{~m}$ & $\mathrm{~L}$ & depressed & 8 & well & - & - & - \\
\hline 16 & $52 \mathrm{f}$ & $\mathrm{U}$ & depressed & 12 & sig & - & - & - \\
\hline 17 & $49 \mathrm{~m}$ & M & depressed & 23 & sig & - & - & - \\
\hline 18 & $54 \mathrm{~m}$ & $\mathrm{U}$ & depressed & 65 & $\bmod$ & - & - & - \\
\hline 19 & $72 \mathrm{f}$ & $\mathrm{L}$ & elevated & 18 & well & - & - & - \\
\hline 20 & $60 \mathrm{f}$ & $\mathrm{L}$ & depressed & 20 & well & - & - & - \\
\hline 21 & $59 \mathrm{~m}$ & $\mathrm{~L}$ & depressed & 38 & sig & - & - & - \\
\hline 22 & $81 \mathrm{~m}$ & M & depressed & 15 & sig & - & - & - \\
\hline Total & & & & & & $3 / 22$ & $4 / 22$ & $7 / 22$ \\
\hline Total $\%$ & & & & & & $(13.6 \%)$ & $(18.2 \%)$ & $(31.8 \%)$ \\
\hline
\end{tabular}

$\mathrm{U}$, upper third of stomach; M, middle third of stomach; L, lower third of stomach; sig, signet ring cell carcinoma.

poorly differentiated adenocarcinomas. TFF1 was expressed in 17 of 20 differentiated carcinomas, 7 of 7 signet ring cell carcinomas, and 6 of 6 poorly differentiated adenocarcinomas. MUC2 and TFF1 were expressed in 5 of $13(38.5 \%)$ and 13 of $13(100 \%)$ undifferentiated carcinomas (poorly differentiated adenocarcinoma and signet ring cell carcinoma), respectively. The positive rate of TFF1 was significantly higher than that of MUC2 in the undifferentiated carcinoma specimens $(\mathrm{p}=0.002)$ (Table I).

Genetic detection of lymph node micrometastases. MUC2 was expressed in 15 of 310 lymph nodes (4.8\%) and 6 of 33 patients $(18.2 \%)$. TFF1 was expressed in 9 of 310 lymph nodes $(2.9 \%)$ and 6 of 33 patients $(18.2 \%)$. The detection rates of both markers were not statistically significant. However, the detection rate of LMMs increased to 6.8\% (21 lymph nodes) and $33.3 \%$ (11 patients) by using the duplex RT-PCR with the MUC2 and TFF1 assay.

Duplex marker expression and lymph node micrometastases in mucosal cancer (Table II). Fourteen of 22 tumor specimens (63.6\%) were positive for MUC2 and 10 of 12 differentiated carcinoma specimens (83.3\%) were positive for MUC2. Only 4 of 10 undifferentiated carcinoma specimens (40\%), however, were positive for MUC2. 20 of 22 tumor specimens (90.9\%) were positive for TFF1 and 10 of 12 differentiated carcinoma specimens $(83.3 \%)$ were positive for TFF1. All undifferentiated carcinoma specimens were positive for TFF1. The positive rate of TFF1 in undifferentiated cancer was significantly higher than that of MUC2 $(\mathrm{p}=0.011)$. All carcinoma specimens were positive for MUC2 and/or TFF1.

In the cases of mucosal cancer, we detected LMMs in 3 of 14 patients $(21.4 \%)$ and 3 of 131 lymph nodes $(2.3 \%)$ using MUC2 RT-PCR assay. Two of 3 such cases were differentiated cancer, and the other case was undifferentiated cancer. By using the TFF1 RT-PCR assay, we detected LMMs in 4 of 20 patients (20\%) and 5 of 188 lymph nodes $(2.7 \%)$. Three of 4 such cases were undifferentiated cancer, while the remaining case was differentiated cancer. We detected LMMs in 7 of 22 patients $(31.8 \%)$ and 9 of 208 lymph nodes $(4.3 \%)$ by using the duplex RT-PCR with the MUC2 and TFF1 assay. Each MUC2-positive case was negative for TFF1 and each TFF1-positive case was negative for MUC2. Represen-tative results are shown in Fig. 1. The 
(a)

Pt.4

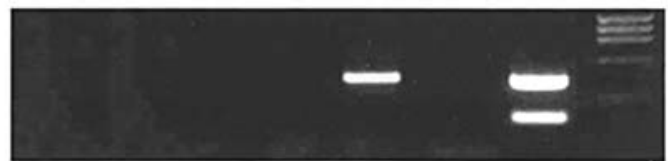

$\begin{array}{llllll}7 & 6 & 4 \mathrm{~d} & 3 & 1 & \mathrm{Ca}\end{array}$
TFF1 438bp

MUC2 189bp

(b)

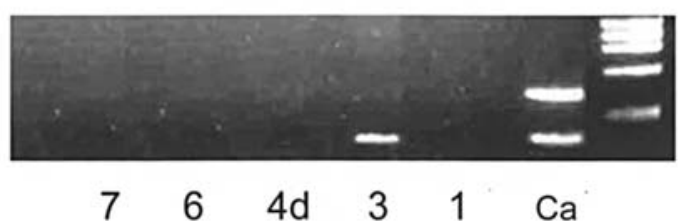

TFF1 438bp

MUC2 189bp

$\begin{array}{lllllll}7 & 6 & 4 \mathrm{~d} & 3 & 1 & \mathrm{Ca}\end{array}$

Figure 1. (a) Representative results for a TFF1-positive/MUC2-negative case. (b) A TFF1-negative/MUC2-positive case. Ca, primary carcinoma sample 1-7. Classification numbers were assigned to lymph nodes according to the Japanese Gastric Cancer Association criteria.

detection rate of the duplex RT-PCR assay was greater, although not significantly, than that of MUC2 or TFF1 alone.

In mucosal cancer, 6 of 7 LMM-positive cases were depressed-type carcinoma. The remaining one case (patient 7) had elevated-type carcinoma. This last case had previously experienced EMR. All primary cancer cases were of the depressed-type.

Correlation between lymph node micrometastases and clinicopathological findings in mucosal depressed cancer (Table III). The incidence of LMMs was significantly higher in patients with large tumors $(\geq 25 \mathrm{~mm})$ than in patients with small tumors $(<25 \mathrm{~mm})(\mathrm{p}=0.028)$. Sex, age, tumor location, and histological type did not correlate with the incidence of LMMs.

Lymph node micrometastases in submucosal cancer. We detected micrometastasis in 4 of 11 patients (36.4\%) and 13 of 102 lymph nodes $(12.7 \%)$ using duplex RT-PCR with the MUC2 and TFF1 assay. However, no clinicopathological factors were associated with the incidence of lymph node micrometastasis in submucosal cancer (data not shown).

\section{Discussion}

Patients with EGC generally have excellent prognoses $(33,34)$. Regional lymph node metastasis is the most important prognostic factor for patients with EGC. Therefore, it has been recognized that an excellent prognosis involves a radical gastrectomy with extensive lymphadenectomy. However, in EGC lymph node metastasis comprises up to $4 \%$ of the mucosal type and $\sim 23 \%$ of the submucosal type (1). This indicates that many of the patients with EGC received unnecessary extensive lymphadenectomies. Thus, it is more important to select those patients who do not have lymph node metastatic disease.

LMM is defined as micrometastatic foci that cannot be detected by H\&E staining (7). Micrometastases that exist at the time of the primary tumor operation are thought to be
Table III. The correlation between duplex RT-PCR analysis and clinicopathological findings in depressed-type mucosal cancer patients.

\begin{tabular}{|c|c|c|c|}
\hline Parameter & $\begin{array}{c}\text { RT-PCR } \\
\text { Positive case/ } \\
\text { total }\end{array}$ & Rate $(\%)$ & P-value \\
\hline
\end{tabular}

Tumor size

$\begin{array}{lrrr}<25 \mathrm{~mm} & 0 / 6 & 0.0 & 0.028 \\ \geq 25 \mathrm{~mm} & 6 / 9 & 66.7 & \end{array}$

Tumor location

$\begin{array}{lll}\mathrm{U} & 1 / 3 & 33.3 \\ \mathrm{M} & 3 / 7 & 42.9 \\ \mathrm{~L} & 2 / 5 & 40.0\end{array}$

Histological type

$\begin{array}{lll}\text { Differentiated } & 2 / 6 & 33.3 \\ \text { Undifferentiated } & 4 / 9 & 44.4\end{array}$

Sex

\begin{tabular}{llll} 
Male & $4 / 11$ & 36.4 & 1.00 \\
Female & $2 / 4$ & 50.0 & \\
Age & & & \\
$<60 \mathrm{yr}$ & $3 / 9$ & 33.3 & 0.62 \\
$\geq 60 \mathrm{yr}$ & $3 / 6$ & 50.0 & \\
\hline
\end{tabular}

$\mathrm{U}$, upper third of stomach; M, middle third of stomach; L, lower third of stomach; differentiated, well and moderately differentiated adenocarcinoma; undifferentiated, poorly differentiated adenocarcinoma and signet ring cell carcinoma.

associated with tumor recurrence in $\operatorname{EGC}(8,9)$. We previously reported that MUC2 is a useful marker for detecting LMM in gastric cancer (27). However, MUC2 was rarely expressed in early, poorly differentiated adeno- 
carcinomas. To improve the detection rate, we sought another target gene which is expressed in early, poorly differentiated gastric cancer cells.

TFF1 belongs to the trefoil factor family (TFF), which is a group of small molecule polypeptides secreted mainly by gastrointestinal mucous cells. TFF1 is immunohistochemically detected in $66.7 \%$ of gastric carcinoma cases. A significant association between immunohistochemical TFF1 expression and diffuse-type histological classification has been revealed (30). In our study, the positive rate of TFF1 was significantly higher than that of MUC2 in the undifferentiated carcinoma specimens $(\mathrm{p}=0.002)$ (Table I). All carcinoma specimens were positive for MUC2 and/or TFF1.

CEA mRNA is a target molecule used in the detection of micrometastases in clinical trials of gastric cancer patients (7, 10-13). However, we previously revealed that MUC2 is a more useful marker for detecting lymph node micrometastases than CEA, especially in early gastric cancer (27). In this study, we reported that the detection rate of duplex RT-PCR with MUC2 and TFF1 is higher, although not significantly, than that of MUC2 or TFF1 alone, suggesting that it is a more useful method of LMM detection in EGC.

We detected LMMs in 7 of 22 patients (31.8\%), especially in mucosal cancer. Of the 7 cases, 3 were MUC2-positive/ TFF1-negative and the other 4 were MUC2-negative/TFF1positive. There were no cases, however, where both markers were positive, suggesting that single markers are inadequate. Thus, duplex RT-PCR with MUC2 and TFF1 is necessary to detect LMM in mucosal gastric cancer.

In Japan, the accepted indications for EMR have been: (i) well-differentiated elevated lesions $<20 \mathrm{~mm}$ in size, and (ii) small $(\leq 10 \mathrm{~mm})$ depressed well-differentiated tumors without ulceration (31). These criteria are rather strict, and many patients may be subject to unnecessary surgery. Therefore, the indications for EMR have been gradually broadened. A large case series has suggested that patients with tumors $<30 \mathrm{~mm}$ without ulceration, regardless of the histological type, are appropriate candidates for EMR (32).

In our study, LMMs were detected only in depressed cancers with the exception of one case, recalling that that one case had previous EMR. Limited to primary cancer, LMMs were not detected in elevated-type cancer with a 20-30-mm diameter. Therefore, our data supports previous reports. In depressed-type cancer, the incidence of LMMs was significantly higher in patients with large tumors $(\geq 25 \mathrm{~mm})$ than in patients with small tumors $(<25 \mathrm{~mm})(\mathrm{p}=0.028)$ (Table III). According to our study, it will be possible to broaden the indications for EMR in elevated-type cancer.

The follow-up period ranged from 18 to 42 months with a median of 24 months, and currently all patients are alive. There is no correlation between the incidence of LMM and short-term survival. However, long-term follow-up studies are required to evaluate its clinical significance.

Furthermore, there was no correlation between the incidence of LMMs and the clinicopathological findings in submucosal cancer patients. It proved difficult to predict lymph node metastatic disease in submucosal gastric cancer. Thus, gastrectomy with regional lymph node dissection is necessary for such patients.
The duplex RT-PCR assay using both MUC2 and TFF1 provides higher LMM detection rates than either MUC2 or TFF1 alone, especially for mucosal gastric cancer. LMM detected by this method may prove useful in broadening the indications for EMR in elevated-type cancer.

\section{References}

1. Hohenberger P and Gretschel S: Gastric cancer. Lancet 362: 305-315, 2003.

2. Yamamoto H, Yube T, Isoda N, et al: A novel method of endoscopic mucosal resection using sodium hyaluronate. Gastrointest Endosc 50: 251-256, 1999.

3. Ono H, Kondo H, Gotoda T, et al: Endoscopic mucosal resection for treatment of early gastric cancer. Gut 248: 225-229, 2001.

4. Ohkuwa M, Hosokawa K, Boku N, et al: New endoscopic treatment for intramucosal gastric tumors using an insulated-tip diathermic knife. Endoscopy 33: 221-226, 2001

5. Miyamoto S, Muto M, Hamamoto Y, et al: A new technique for endoscopic mucosal resection with an insulated-tip electrosurgical knife improves the completeness of resection of intramucosal gastric neoplasms. Gastrointest Endosc 55: 576-581, 2002.

6. Yamamoto H, Kawata H, Sunada K, et al: Success rate of curative endoscopic mucosal resection with circumferential mucosal incision assisted by submucosal injection of sodium hyaluronate. Gastrointest Endosc 56: 507-512, 2002.

7. Matsumoto M, Natsugoe S, Ishigami S, et al: Lymph node micrometastasis and lymphatic mapping determined by reverse transcriptase-polymerase chain reaction in $\mathrm{pN0}$ gastric carcinoma. Surgery 131: 630-635, 2002.

8. Baba H, Maehara Y, Takeuchi H, et al: Effect of lymph node dissection on the prognosis in patients with node-negative early gastric cancer. Surgery 117: 165-169, 1994.

9. Maehara Y, Oshiro T, Endo K, et al: Clinical significance of occult micrometastasis in lymph nodes from patients with early gastric cancer who died of recurrence. Surgery 119: 397-402, 1996.

10. Okada Y, Fujiwara Y, Yamamoto H, et al: Genetic detection of lymph node micrometastases in patients with gastric carcinoma by multiple-marker reverse transcriptase-polymerase chain reaction assay. Cancer 92: 2056-2064, 2001.

11. Mori M, Mimori K, Inoue H, et al: Detection of cancer micrometastases in lymph nodes by reverse transcriptasepolymerase chain reaction. Cancer Res 55: 3417-3420, 1995.

12. Mori M, Mimori K, Ueo H, et al: Clinical significance of molecular detection of carcinoma cells in lymph nodes and peripheral blood by reverse transcriptase-polymerase chain reaction in patients with gastrointestinal or breast carcinomas. $\mathbf{J}$ Clin Oncol 16: 128-132, 1998.

13. Kubota K, Nakanishi H, Hiki N, et al: Quantitative detection of micrometastases in the lymph nodes of gastric cancer patients with real-time RT-PCR: A comparative study with immunohistochemistry. Int J Cancer 105: 136-143, 2003.

14. Noguchi S, Hiratsuka M, Furukawa H, et al: Detection of gastric cancer micrometastases in lymph nodes by amplification of keratin 19 mRNA with reverse transcriptase-polymerase chain reaction. Jpn J Cancer Res 87: 650-654, 1996.

15. Futamura M, Takagi Y, Koumura H, et al: Spread of colorectal cancer micrometastases in regional lymph nodes by reverse transcriptase-polymerase chain reactions for carcinoembryonic antigen and cytokeratin 20. J Surg Oncol 68: 34-40, 1998.

16. Miyake Y, Yamamoto H, Fujiwara Y, et al: Extensive micrometastases to lymph nodes as a marker for rapid recurrence of colorectal cancer: a study of lymphatic mapping. Clin Cancer Res 7: 1350-1357, 2001.

17. Noura S, Yamamoto H, Ohnishi T, et al: Comparative detection of lymph node micrometastases of stage II colorectal cancer by reverse transcriptase-polymerase chain reaction and immunohistochemistry. J Clin Oncol 20: 4232-4241, 2002.

18. Gunn J, McCall JL, Yun K, et al: Detection of cancer micrometastases in colorectal cancer patients by K19 and K20 reverse transcriptase-polymerase chain reaction. Lab Invest 75: 611-616, 1996.

19. Dorudi S, Kinrade E, Marshall NC, et al: Genetic detection of lymph node micrometastases in patients with colorectal cancer. Br J Surg 85: 98-100, 1998

20. Weitz J, Kienle P, Magener A, et al: Detection of disseminated colorectal cancer cells in lymph nodes, blood and bone marrow. Clin Cancer Res 5: 1830-1836, 1999. 
21. Merrie AE, Yun K, van Rij AM, et al: Detection and significance of minimal residual disease in colorectal cancer. Histol Histopathol 14: 561-569, 1999.

22. Bostick PJ, Chatterjee S, Chi DD, et al: Limitation of specific reverse-transcriptase polymerase chain reaction markers in the detection of metastases in the lymph nodes and blood of breast cancer patients. J Clin Oncol 16: 2632-2640, 1998.

23. Ko Y, Klinz M, Totzke G, et al: Limitation of the reverse transcriptase-polymerase chain reaction method for the detection of carcinoembryonic antigen-positive tumor cells in peripheral blood. Clin Cancer Res 4: 141-146, 1998.

24. Castells A, Boix L, Bessa X, et al: Detection of colonic cells in peripheral blood of colorectal cancer patients by means of reverse transcriptase and polymerase chain reaction. Br J Cancer 78: 1368-1372, 1998.

25. Zippelius A, Kufer P, Honold G, et al: Limitation of reversetranscriptase polymerase chain reaction analyses for detection of micrometastatic epithelial cancer cells in bone marrow. J Clin Oncol 15: 2701-2708, 1997.

26. Champelovier P, Mongelard F and Seigneurin D: CK20 gene expression: technical limits for detection of circulating tumor cells. Anticancer Res 19: 2073-2078, 1999.

27. Sonoda H, Yamamoto K, Kushima R, et al: Detection of lymph node micrometastasis in gastric cancer by MUC2 RT-PCR: Usefulness in pT1 cases. J Surg Oncol 88: 63-70, 2004.
28. Katoh M: Trefoil factors and human gastric cancer (Review). Int J Mol Med 12: 3-9, 2003.

29. Japanese Gastric Cancer Association: Japanese classification of gastric carcinoma. Gastric cancer 1: 10-24, 1998.

30. Machado JC, Nogueira AM, Carneiro F, et al: Gastric carcinoma exhibits distinct types of cell differentiation: an immunohistochemical study of trefoil peptides (TFF1 and TFF2) and mucins (MUC1, MUC2, MUC5AC, and MUC6). J Pathol 190: 437-443, 2000.

31. Tsujitani S, Oka S, Saito H, et al: Less invasive surgery for early gastric cancer based on the low probability of lymph node metastasis. Surgery 125: 148-154, 1999.

32. Gotoda T, Yanagisawa A, Sasako M, et al: Incidence of lymph node metastasis from early gastric cancer: estimation with a large number of cases at two large centers. Gastric cancer 3: $219-225,2000$.

33. Namieno T, Koito K, Higashi T, et al: General pattern of lymph node metastasis in early gastric carcinoma. World J Surg 20: 996-1000, 1996.

34. Nakamura K, Ueyama T, Yao T, et al: Pathology and prognosis of gastric carcinoma: findings in 10,000 patients who underwent primary gastrectomy. Cancer 70: 1030-1037, 1992. 